\title{
Österreichs Kirchenpresse auf der Suche nach ihrem Markt
}

\author{
von Maximilian Gottschlich
}

Für die kirchliche Presse in Ơsterreich - allerdings nicht nur hier - sind viele Leser bereits gestorben. Und das im doppelten Sinn des Wortes. In der sich ohnehin nicht durch üppige Vielfalt auszeichnenden Medienlandschaft eines zu fast $90 \%$ katholischen Landes macht sich die kircheneigene Presse kaum bemerkbar.

Dort, wo sie sich gesellschaftspolitisch engagiert - etwa in der Frage der Fristenlösung -, engagiert sie sich unter Ausschluß jener katholischen Offentlichkeit, die gerade der Argumente zur Meinungsbildung und Entscheidungshilfe bedarf; dort, wo die Kirchenpresse im traditionellen Stil als Organ der Diözese Informationen weitergibt, informiert sie Mitglieder, deren Orientierungsbedürfnis sich nicht, oder nur am Rande, auf (amtliche) Strukturen und interne Organisation bezieht, sondern primär auf die Fragen nach Sinn und Sinnerfüllung; dort, wo kirchliche Publizistik nach Wesen und Erscheinung fragt, also in Anspruch nimmt, Sinn herzustellen, enttäuscht sie vielfach durch mangelnde Uberzeugungskraft, durch mangelnde Praktikabilität im Nachvollzug, enttäuscht sie vor allem aber auch durch die Unfähigkeit, Atmosphäre zu schaffen, mit „der Ebene des Rationalen und Zweckhaften" ${ }^{1}$ zugleich auch Gefühl zu vermitteln.

Angesichts eines vorhersehbaren „biologischen Endes“2 der kirchlichen Presse in Ossterreich, läßt sich in der Tat fragen, ob ihr redaktionelles Programm ausreicht, dem optimistisch deklarierten - wenngleich auch nicht weiter operationalisierten Selbstverständnis als „Kommunikations- und Informationsorgane der Diözesen “3 auch zu entsprechen.

Die Gesamtauflage der elf Kirchenzeitungen Ósterreichs hält (noch) bei 470.000; das ist ein Auflagenrückgang innerhalb der letzten zehn Jahre um rund $10 \% 0^{4}$.

\section{Selbsthilfe einer Kirchenzeitung}

Beschluß 89 des Österreichischen Synodalen Vorgangs (Kapitel: Kirche und Massenmedien) hält die Notwendigkeit fest, „ein Pressekonzept der Kirche auszuarbeiten...“, das „... den Bestand, die Möglichkeiten und die anzustrebenden Ziele in einer Spannweite von gesamtösterreichischen Organen bis zu den Pfarrblättern, einschließlich der Ordensschriften, erfassen, ordnen und aufeinander abstimmen [soll], um Konzentrations- und Ausbautrends und einen Prioritätenkatalog festlegen zu können "s.

Die empirischen Grundlagen zur Erstellung eines solchen Konzepts fehlen allerdings noch weitgehend: Die Mehrzahl der Studien zur religiösen Situation der Ósterreicher klammern die Leserschaftsforschung völlig aus - der Bereich Kirche und kirchliche Publizistik, Kirchlichkeit und Mediennutzung, zählt bislang noch nicht zum Feld kirchlicher Sozialforschung.

Mit der ersten Leserbefragung in der Geschichte der kirchlichen Presse in Osterreich griff das Organ der Diözese Wien, die "Wiener Kirchenzeitung“, zur Selbsthilfe und

Dr. Maximilian Gottschlich ist Hochschul-Assistent am Institut für Publizistik an der Universität Wien. 
trug damit der Erkenntnis Rechnung, daß es an der Zeit sei, den eigenen Standort unter den gegebenen Bedingungen neu zu bestimmen, dem (noch verbliebenen) Leser das Wort zu geben ${ }^{6}$.

Zum Zeitpunkt der Befragung (Mai 1974) betrug die gedruckte Auflage der „Wiener Kirchenzeitung" 67.216 - um rund $12 \%$ weniger als im Vergleichsmonat des Jahres 1972. Als (äußere) Gründe für diesen Rückgang wurden vor allem genannt:

a) Schwund der Kirchlichkeit in der Bevölkerung (Rückgang des Meßbesuchs etwa in Wien-Stadt auf rund $9 \%)^{7}$;

b) Uberalterung der Leserstruktur (wobei es nicht gelingt, die Jugend und die etwa 30-45jährigen anzusprechen ${ }^{8}$;

c) die Polarisierung innerhalb der Katholiken (wobei es nicht gelingt, redaktionell sowohl den "Traditionalisten" als auch den "Progressisten" zu entsprechen und damit der weitgehenden Ablehnung sowohl durch die einen, als auch durch die anderen zu entgehen).

Zur „journalistischen Gewissenserforschung “, also zur Beantwortung der Frage, inwieweit die Ursachen des publizistischen Mißerfolgs in der redaktionellen Konzeption des Blattes selbst zu suchen sei, sollten die Leser antworten zu Schreibweise, Gestaltung und Format der Zeitung, sowie zu den angebotenen Inhalten dienen. $\mathrm{Zu}$ zwei offenen Fragen (,in der Kirchenzeitung vermisse ich" und „in der Kirchenzeitung gefällt mir nicht ${ }^{*}$ ) sollten die Leser frei ihre Meinung äußern. Den im Blattinneren abgedruckten Fragebogen sandten $3.373(5,2 \%)$ Leser der Zeitung zurück (von ihnen gaben mehr als $80 \%$ an, die Kirchenzeitung über ein pfarrinternes Verteilersystem bzw. durch Kauf an der Kirchentür zu erwerben, rund $11 \%$ der Einsender beziehen das Blatt über ein Abonnement).

Die Antworten wurden gegeben, die Preise für das "Mittun" verlost - offen blieb für Verlag und Redaktion die Frage, ob diese Antworten mehr zur Freude Anlaß geben oder mehr zur Resignation ob des Ausbleibens des erwarteten (richtungsweisenden weichenstellenden) Knalleffekts.

\section{Ergebnis der Aktion ${ }^{9}$}

Die Mehrheit der antwortwilligen Stammleser beurteilt die Schreibweise des Blatts als "gut verständlich" (72,6\% der eingesandten Antworten), ihnen gefällt die äußere Aufmachung (61,8\%), und sie finden, daß das Format "so bleiben soll, wie bisher" $(64,3 \%)$ - knapp ein Drittel $(31,4 \%)$ hätte Kirchliches lieber auf Kleinformat.

Für eine - zur Diskussion gestellte - Beibehaltung der Kinderseite und regelmäßige Jugendbeiträge - schwache Stelle einer Redaktion, die in der Regel für Leser zu schreiben gewohnt ist, deren Alter zu einem hohen Prozentsatz über 50 Jahren liegt -, plädierten allerdings mehr als $80 \%$ der Einsender (von ihnen gaben 37,4\% ihr Alter $z$ wischen 46 und 65 Jahren, 28,5\% eines über 65 Jahren, aber nur 25,2\% ihr Alter zwischen 26 und 45 Jahren an; nur 7,5\% waren jünger als 25 Jahre). Von wenigen Ausnahmen abgesehen sind die Einsender des Fragebogens (22,9\% Angestellte, 22,6\% Pensionäre, 20,1\% Hausfrauen, 7,1\% Landwirte, 4,4\% Priester und Ordensleute, 4,2\% Schüler und Lehrlinge, 2,7\% Arbeiter und 1,7\% freiberuflich Tätige) mit dem inhaltlichen Angebot der „Wiener Kirchenzeitung“ zufrieden. Um nur einige Ergebnisse herauszugreifen: 
Die "religiös-kirchlichen Artikel, die auf Seite 1 stehen“, werden von $72,7 \%$ "oft" gelesen, die „Berichte über Kirche in Osterreich" von 76,8\%. Nach ihren Angaben lesen mehr als zwei Drittel (69,0\%) „oft“ die „Berichte aus der Diözese“, für die „Berichte aus Dekanaten und Pfarren" finden sich auch 61,1\% interessierte Leser. $60,2 \%$ geben an, "die gesellschaftspolitischen Artikel, die auf Seite 1 stehen ", "oft" zu lesen, während die "Ankündigungen von Veranstaltungen" vergleichsweise auf weniger Gegenliebe stoßen (45\% lesen diese Mitteilungen „oft“, 33,5\% "gelegentlich" und 15,4\% „fast nie“).

Ebenso finden „längere, ausführliche Kommentare“, Buchbesprechungen, Kreuzworträtsel, Kurzgeschichten und Romane eher wenig Interesse beim Leser. Auch die Quantität des redaktionellen Angebots scheint im richtigen Maß dosiert zu sein: mehrheitlich stimmten die Befragten für eine thematische Gewichtung "wie bisher". $28,2 \%$ plädierten für ein "mehr" an "Berichten und Nachrichten aus der Kirche in Osterreich", ein Drittel der Leser (33,8\%) äußerte den Wunsch nach "mehr" Bejträgen $\mathrm{zu}$ "Lebensfragen aus dem Bereich der Familie und Erziehung “. Bei rund $26 \%$ der Leser besteht vermehrter Bedarf an „Berichten und Nachrichten aus Dekanaten und Pfarren“, sowie an der Behandlung von "Lebensfragen aus Beruf (Schule) und Freizeit". Trotz "kopflastiger Alterspyramide ${ }^{\star 10}$ reicht den meisten Lesern $(66,6 \%)$ das Angebot an "Lebensfragen für alte Menschen", 24,3\% hä̈ten gerne "mehr" zu diesem Thema. Die Ablehnung einzelner angebotener Inhalte hält sich in Grenzen: 13,5\% wollen die "Erbaulichen, spirituellen Beiträge" reduziert wissen, 3,7\% wollen Kontemplatives "gar nicht“. Für ein "weniger" an "gesellschaftspolitischen Artikeln" stimmten 12,5\% der solcherart zur Mitbestimmung aufgeforderten Leser.

Resümee: 3.373 Leser der „Wiener Kirchenzeitung" sind mehrheitlich mit dem Blatt zufrieden. Dennoch - oder gerade deswegen - gibt die „Do it yourself“-Untersuchung des Presseorgans der Diözese Wien Anlaß zu einigen grundsätzlichen Erwägungen, vor allem was die angewandte Methode und die Relevanz der Ergebnisse betrifft.

\section{Die Falschen befragt}

In signifikanter Weise spiegelt die Untersuchung ein zweifaches Mißverständnis wider, das bei den kirchlichen Kommunikatoren derzeit noch vorzuherrschen scheint: zum einen nämlich eine weitgehende Fehleinschätzung der Möglichkeiten, den Rezipienten "wieder einzuholen", - also eine Fehleinschätzung der Korrigierbarkeit eigenen publizistischen Fehlverhaltens durch sporadische Aktivitäten, aufgrund derer man meint, sich - wenn schon nicht anders, so doch zumindest der Optik nach erfolgreich an eine vorauseilende Medienentwicklung "anhängen“ zu können; zum anderen die - historisch aus dem belasteten Verhältnis von Theologie und Soziologie gewachsene - Annahme, auf dem Niveau gewohnter (sicherlich nützlicher) sozialstatistischer Datenerhebung allein, ausreichend Aufschluß über den Leser bzw. Nichtleser als Zielobjekt kommunikativen Bemühens zu erhalten.

Die proklamierte Neuorientierung der kirchlichen Presse wird in Zukunft primär auf der Basis einer qualitativen Leserschaftsforschung, wie sie mehr und mehr in erster Linie zur Qualifizierung eines publizistischen Produkts als Werbeträger von 
den Zeitungsverlagen und Publikumszeitschriften betrieben wird, zu erfolgen haben. Empirische Studien im Bereich kirchlicher Publizistik müßten in Hinsicht auf flexible redaktionelle Konzeption vor allem Auskunft geben über das Leseverhalten sowohl der Intensivchristen als auch der Randschichten, über die Intensität der Leser-BlattBindung und das Image der Kirchenpresse.

So hätte, - um beim Beispiel der Untersuchung der „Wiener Kirchenzeitung“ zu bleiben, - die Strategie einer derartigen Erhebung andere Schwerpunkte setzen müssen:

- Weniger die Stamm- als vielmehr die Grenzleser und jene, die die Kirchenzeitung bereits abbestellt haben, hätten untersucht werden sollen.

- Signifikantere und vor allem zuverlässigere Ergebnisse, als die in einem Fragebogen konzentrierten Fragen nach der Häufigkeit des Lesens bestimmter, vorcodierter Inhalte („kirchenpolitische Beiträge“), hätte der in der Zeitungsbranche erfolgreich verwendete redaktionelle Copy-Test ergeben. Dieses, bei einem repräsentativen Querschnitt der einzelnen Leserschichten anzuwendende Verfahren, verhindert weitgehend jene mögliche Verzerrung der Ergebnisse, die dadurch zustande kommen kann, daß die in einem Fragebogen üblicherweise vorgesehene Zuordnung der angegebenen Themen zu den jeweiligen Skalenwerten ("häufig" - "gelegentlich" — „fast nie“) eher danach vorgenommen wird, welche Inhalte ihrer thematischen Nähe wegen auf die Kirchenzeitung zu passen scheinen, und nicht danach, in welchem Ausmaß man tatsächlich die entsprechenden Artikel liest ${ }^{11}$. Der Copy-Test ist in der Regel in eine qualitative Leserbefragung (Projektive Tests, Polaritätsprofil) eingebunden. Methodenimmanente Mängel der Frageaktion:

- Gerade die eben erwähnte Skalierung („oft" — „gelegentlich“ — „fast nie“) bei der Frage: Was lesen Sie in der Kirchenzeitung? erscheint zu wenig valid; ebenso gibt die Skalierung "wie bisher" - "mehr" - „weniger" — "gar nicht" bei der Frage: Was möchten Sie in der Kirchenzeitung haben? keine Auskunft beispielsweise darüber, zu welchen Ungunsten ein „mehr als bisher" eines Themenbereichs ausfallen sollte.

- Bei den offenen Fragen („In der Kirchenzeitung vermisse ich“ und „In der Kirchenzeitung gefällt mir nicht ${ }^{\text {") }}$ ) wären zusätzliche Hinweise gerade auch aus einer Begründung der gegebenen Antworten zu erwarten gewesen - die Aufforderung dazu fehite allerdings.

- Auch die Fragen zur Sozialstatistik hätten einer stärkeren Differenzierung

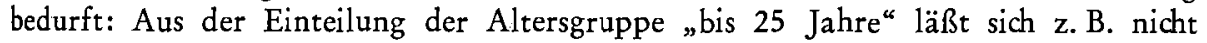
ablesen, wie die 14-16jährigen etwa zur Kirchenzeitung stehen. Ebenso verspricht beim soziographischen Merkmal „Berufsstand“ die Angabe „in Ausbildung (Schule oder Lehre)" nur bedingt verwertbare Ergebnisse: für den katholischen Schüler werden andere Kriterien einer möglichen Leser-Blatt-Bindung, werden andere Nutzungsgewohnheiten ausschlaggebend sein, als für den katholischen Lehrling.

- Geht man davon aus, daß das Image einer Kirchenzeitung bei den Pfarrmitgliedern von der Kommunikationssituation der Pfarre, aber auch vom Verhalten des Pfarrers gegenüber den verschiedenen Ausprägungen kirchlicher Presse mitgeprägt wird, daß daran bisweilen selbst Fragen der Organisation des Vertriebs im lokalen Raum hängen, dann wäre die Kenntnis der Pfarrzugehörigkeit der Einsender sicher von einigem Interesse gewesen. 


\section{Auf dem Weg zum Kommunikationsmarketing}

Methode und Ergebnisse der ersten Leserumfrage innerhalb der kirchlichen Presse in Osterreich macht es deutlich: Um die Fülle der guten Worte, die prinzipiell bereits vollzogenen Einsichten in die Notwendigkeiten und Bedingungen zukunftsorientierter und zukunftsorientierender Publizistik im konkreten Handeln einzulösen, bedarf es einer der Problematik angemessenen Bereitschaft, in eine systematische Erkenntnisgewinnung auf diesem Gebiet einzutreten. Die Kirche wird die Erforschung des kirchliche Mitgliedschaft mehr oder weniger realisierenden Medienkonsumenten konsequent vorantreiben müssen, sie wird, kurz gesagt, Kommunikationsmarketing betreiben müssen. Der Markt, nämlich das Kirchenvolk, läßt sich heute nicht mehr durch die Merkmale institutionalisierter Bindung an die Orts- oder Diözesankirche beschreiben. Mit den gesamtgesellschaftlichen Kommunikationsstrukturen, der Pluralität des Informationsangebots ${ }^{12}$ und mit einem sich zunehmend differenzierenden Selektionsverhalten der Rezipienten haben sich die Informationsbedürfnisse auch der aktiven Katholiken geändert. Dazu kommt, daß die kirchliche Presse längst kein „Veröffentlichungsmonopol“ mehr für kirchliche Information hat - im Gegenteil: der Eindruck, die Amtskirche verlasse sich bei ihrer Informationstätigkeit lieber auf die „Veröffentlichungsleistung ${ }^{{ }_{13}{ }^{13}}$ weltlicher Informationsmittler, dürf te seine Richtigkeit nicht nur für die Bundesrepublik, sondern auch für Osterreich haben.

Für das Kirchenvolk ist Kirchenpresse nicht mehr und nicht weniger als „eine besondere Erscheinungsform des modernen Massenmediums Presse mit eigener Sachgesetzlichkeit ${ }^{\text {"14 }}$. Kriterium dieser Sachgesetzlichkeit ist vor allem die aus der Funktion von Kirchenpresse resultierende und manifeste Gebundenheit an die Institution Kirche, also die Belastung durch ein weitverbreitetes und tief wurzelndes Image,

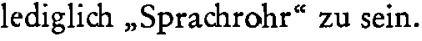

Die bisherigen Praktiken journalistischer Orientierung an den Lesern reichen heute nicht mehr aus, die Existenz - nicht nur die materielle Existenz - der Kirchenzeitungen zu sichern. Im sorgfältig geplanten Redaktionsmarketing bietet sich die Möglichkeit, die Konsequenzen zu ziehen und die "Plausibilitätsannahmen “15 über den katholischen Leser und die Informationsbedürfnisse einer innerkirchlichen Offentlichkeit systematisch auf ihren realen Gehalt, auf ihre Tragfähigkeit hin zu überprüfen.

Es stimmt schon: Marketing hat mit Konsum und Absatz zu tun; der Publizitätsverlust der Kirche und ihrer Presse müßte, wie es scheint, Argument genug für diese Kirche als Produzent sein, den Markt, nämlich die Konsumenten kirchlicher Information, kennenzulernen, um der Kirchenpresse - um also dem eigenen, die Institution repräsentierenden Produkt, größtmögliche Erfolgschancen unter den anderen Anbietern und Angeboten am Kommunikationsmarkt mitzugeben.

Es wäre bei der Anwendung der verschiedenen Techniken der Rezipientenforschung verfehlt, die Fragen der kirchlichen Publizistik als Einzelprobleme zu sehen. Rezipientenforschung und Kommunikationsmarketing im kirchlich-religiösen Bereich wind sich immer auch zugleich mit der Aufgabe konfrontiert sehen müssen, die übergreifende "Sphäre des Glaubens"16 in den Prozeß der Erkenntnisgewinnung miteinzubeziehen - wenngleich diesem Bemühen auch Grenzen gesetzt sind. So wird es bei der Leserschaftsforschung in Zukunft speziell auch darum gehen müssen, systematisch Informationen zu sammeln, etwa - um nur einige wenige Fragenkomplexe anzuführen - über 
- die Einstellung der Zielgruppe zum christlichen Glauben (Glaubenshindernisse), zur Institution Kirche und zur kircheneigenen Publizistik als Vermittler zwischen Kirche und Kirchenvolk,

- die Selektionskriterien beim Informationskonsum (Intensiv-Christen und Randschichten), inwieweit Transzendenzbezug, Ausmaß und Qualität der religiösen Bedingtheit sozialer Verhaltensweisen und -erwartungen auch vorgeschaltete Selektionskriterien beim täglichen Medienkonsum darstellen,

- Reichweite der Medien und Mediennutzung der Zielgruppe,

- Freizeitverhalten (Kirchenzeitung als „Sonntagszeitung“),

- die Organisation des pfarrinternen Informationsflusses (Pfarrbewußtsein), schließlich auch

- die Motivation des Erwerbs (Ablehnung) einer Kirchenzeitung.

Die Kirchenpresse wird dann mehr als überkommene Schablonen von Kirchlichkeit anbieten können, wenn sie, angesichts der kollektiven Bewußtseinssteuerung der weltlichen Medien, flexible Programme entwickelt, um sich je neu auf die kirchlichreligiöse Situation ihrer Adressaten einzustellen. Redaktionsmarketing und die Methoden der empirischen Leserschaftsforschung sind geeignete Instrumente, den Wunsch, mit Hilfe der Kirchenpresse „Zeugnis für die Kirche und für das Leben der Kirche ${ }^{{ }^{17}}$ zu geben, auch Realität werden zu lassen; man muß sich ihrer nur bedienen.

\section{Anmerkungen:}

1. Georg Moser: Die pastorale Bedeutung der Kirchenpresse, in: CS 7:1974, S. 16.

2. Fritz Csoklich: Der triste Zustand der katholischen Presse, in: „Multimedia - Zeitschrift für kritische Medienarbeit", 13:1974, S. 2.

3. Ostereichischer Synodaler Vorgang - Dokumente, Wien 1974.

4. Osterreichischer Synodaler Vorgang, a. a. O., S. 146.

5. Osterreichischer Synodaler Vorgang, a. a. O., S. 135.

6. Der Fragebogen wurde in der Nummer vom 26. 5. 1974 abgedrudkt.

7. Vgl. dazu Paul M. Zulehner: Verfällt die Kirchlichkeit in Osterreich?, Graz 1971, S. 7.

8. In dieser Altersgruppe sind die häufigsten Kirchenaustritte zu verzeichnen.

9. Die Ergebnisse wurden der „Wiener Kirchenzeitung“ vom 29. September 1974 entnommen.

10. Chefredakteur Anton Fellner in der "Wiener Kirchenzeitung“ vom 29. September 1974, S. 12.

11. Vgl. dazu: Hör Zu. Qualitative Analyse der Nutzungsintensität, Hamburg 1967 (Infratest), S. 90.

12. Vgl. dazu: Peter Glotz / Wolfgang R. Langenbucher: Der mißachtete Leser - zur Kritik der deutschen Presse, Köln-Berlin 1969, S. 146 ff.

13. Michael Schmolke: Kirchenpresse und innerkirchliche Information, in: CS 5:1972, S. 126.

14. Michael Schmolke, a. a. O., S. 124.

15. Peter Glotz / Wolfgang R. Langenbucher, a. a. O., S. 148.

16. René König: Handbuch der empirischen Sozialforschung, 2. Band, Stuttgart 1969, S. 1115.

17. Georg Moser, a. a. O., S. 14. 


\section{S U M M A R Y}

The circulation of the Church papers in Austria is going down more and more. The Vienna archdiocesan weekly, "Wiener Kirchenzeitung" through questionnaires tried to find out the wishes of its readers on the content of the paper. The results showed that the majority of the readers who sent back the questionnaires were content with the paper. But in this kind of survey, one only reaches the regular readers, and not the ones who never, or who seldom buy the periodical. Therefore, no outstanding results could be expected this way. The example shows that only a systematic readership survey and mainly the use of qualitative methods of empirical sociology can give reasonable results. Likewise, church press cannot just trust journalistic experience alone. The communication situation of modern society requires also for the Church a systematically planned communications marketing.

\section{RÉSUMÉ}

La presse religieuse en Autriche perd de son tirage en quantité croissante. Afin de mettre fin á cette évolution, l'organe de presse du diocèse de Vienne, le "Wiener Kirchenzeitung", essaya de sonder les souhaits de ses lecteurs concernant la réalisation et l'offre quant au contenu du journal de l'Eglise à l'aide d'une action de questionnaires. Les résultats montrèrent que les lecteurs qui renvoyèrent les questionnaires sont, en majorité, satisfaits de la feuille. Comme on n'avait atteint, à la vérité, à l'aide de cette forme d'enquête que les lecteurs réguliers, engagés, et non pas ceux qui n'achètent que rarement ou plus du tout le journal, on ne pouvait pas s'attendre à des résultats révélateurs. L'exemple montre que seule une recherche systématique du cercle des lecteurs et, en premier lieu, l'utilisation de méthodes qualitatives de la recherche sociale empirique peuvent livrer des résultats capables d'être mis à profit: la presse religieuse ne peut plus se contenter de l'expérience journalistique seule la situation communicative de la société moderne rend nécessaire le marketing de communication systématiquement planifié.

\section{R ESUMEN}

Desciende progresivamente la tirada de la prensa diocesana en Austria. Para detener este debacle el "Wiener Kirchenzeitung", órgano de la diócesis de Viena, trató, mediante una encuesta, de analizar los deseos de sus lectores sobre la presentación y contenido del rotativo. El resultado mostró que la mayoría de los lectores que rellenaron los formularios de la encuesta están contentos con el periódico. Ahora bien, puesto que con tal clase de encuestas solo se entra en contacto con los lectores habituales del periódico y no con quienes ya no lo compran o lo hacen de vez en cuando, apenas pueden esperarse de ellas resultados concluyentes. El ejemplo demuestra que únicamente un análisis sistemático de la clientela y la utilización de métodos cualitativos de la sociología empírica pueden suministrar datos valorativos: La prensa diocesana no puede confiar únicamente en la experiencia periodística. La situación de la sociedad moderna, en lo que a medios de comunicación social se refiere, hace también necesario para la Iglesia un marketing sistemático y planificado de la comunicación social. 